\title{
BENEFICIOS DE LAS ACTIVIDADES LÚDICAS EN ADULTOS MAYORES
}

AUTORES: Juan Enrique Tomala Santana ${ }^{1}$

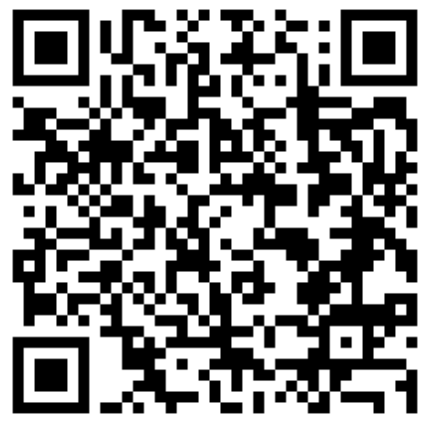

DIRECCIÓN PARA CORRESPONDENCIA: juan.tomalas@ug.edu.ec

Fecha de recepción: 18/01/2020

Fecha de aceptación: 21/03/2020

RESUMEN

El manejo de la lúdica durante el envejecimiento brinda múltiples beneficios al organismo del individuo tanto en el aspecto físico, intelectual y social. Esta investigación tiene como objetivo demostrar que tipos de ejercicios son los adecuados para la rehabilitación de adultos mayores y la prevención de enfermedades crónicas, es un estudio cualitativo basando su estructura en la aplicación de métodos teóricos: inductivo-deductivo, análisis - síntesis, el histórico lógico para estudiar la vinculación y evolución de estas actividades en el proceso activo de los adultos mayores, además los empíricos como: observaciones, encuestas, revisión de documentos para constatar el manejo de las actividades lúdicas dentro del Hogar San José, cuyos resultados fueron favorables afirmando que las técnicas empleadas mediante el uso de la lúdica no solo aportan a la coordinación del cuerpo sino también fomentan valores y la producción de nuevos conocimientos en la población vulnerable.

PALABRAS CLAVE: adulto mayor; actividades lúdicas; vinculación; conocimientos.

\section{BENEFITS OF LEISURE ACTIVITIES IN OLDER ADULTS}

\section{ABSTRACT}

The management of play during aging provides multiple benefits to the body of the individual, both physically, intellectually and socially. This research aims to demonstrate which types of exercises are suitable for the rehabilitation of older adults and the prevention of chronic diseases, it is a qualitative study basing its structure on the application of theoretical hmethods: inductivedeductive, analysis-synthesis, the historical logical to study the linkage and evolution of these activities in the active process of older adults, in addition

${ }^{1}$ Licenciado en ciencias de la comunicación social, Universidad de Guayaquil, Guayaquil 
Juan Enrique Tomala Santana, , ...

empirical ones such as: observations, surveys, document review to verify the management of recreational activities within the Hogar San José, whose results were favorable stating that the techniques used through the use of playfulness not only contribute to the coordination of the body but also promote values and the production of new knowledge in the vulnerable population.

KEYWORDS: older adult; leisure activities; bonding; knowledge.

\section{INTRODUCCIÓN}

A lo largo de la vida el ser humano cumple unas series de etapas determinadas, las cuales generan muchos factores entre ellos el agotamiento al cuerpo y mente del individuo. De acuerdo a datos obtenidos por la Organización mundial de la salud en el año 2015 la población ha envejecido rápidamente pasando de un 12 a $22 \%$; en número absoluto el incremento previsto es de 900 millones a 2000 millones estipulando que "aunque la mayoría de las personas mayores tienen una buena salud, muchas pueden sufrir problemas físicos y mentales" (Organización Mundial de la Salud, 2017).

Los adultos mayores siempre han sido una agrupación social que requiere un cuidado especializado. Según lo establecido en la constitución de la republica de Ecuador son considerados como un grupo de atención prioritaria; cabe recalcar que según estudios efectuados por el INEC durante el 2018 la población de adultos mayores ha presentado un incremento veloz durante los últimos meses, la mayor parte de este círculo poblacional se encuentran aislados en asilos donde reciben una variedad de actividades que sean beneficiosas para el desarrollo integral de su bienestar social.

El envejecimiento adulto ha promovido el uso de la recreación física, factor primordial del movimiento activo del cuerpo humano el cual genera energía a los músculos y cerebro atravesando el proceso lúdico cuyo objetivo es proporcionar un cambio de condición física e intelectual estableciendo equilibrio para que el individuo alcance la calidad de vida adecuada; "la organización de espacios de recreación para una determinada comunidad es una tarea importante a través de ella se contribuye a una vida plena, alegre y satisfactoria" (Querales, 2011).

En la ciudad de Guayaquil existen diversos los lugares de protección que se encargan del cuidado de adultos mayores contando con equipos profesionales capacitados a realizar unas series de rutinas lúdicas a los pacientes las cuales permiten el perfeccionamiento de habilidades y competencias al desarrollo personal y emocional. Por lo tanto, a través de esta investigación se pretende dar a conocer aquellos beneficios obtenidos por la ejecución de las actividades lúdicas en los adultos mayores tomando como población de estudio a las personas de tercera edad del hogar San José.

Es importante especificar; cuando el ser humano pasa a la etapa del envejecimiento su actividad corporal y cognitiva no será igual a una persona joven, por ello con el pasar del tiempo los ejercicios no solo se han convertido en herramienta primordial del cuidado personal sino también en una pieza que establece vínculos sociales con otras personas que comparten un mismo interés. El autor del presente proyecto para facilitar el entendimiento del significado de actividades lúdicas indica la definición del término como "aquello que se puede realizar en el tiempo libre con el objetivo de liberar tensiones, mejorar equilibrio, flexibilidad estimulando agilidad mental en el cerebro" (Lúdico, 2019). 
Durante la tercera edad, el hombre debe poseer un modo de vida óptimo pues; para lograr cuyo propósito de manera positiva y constructiva "el anciano debe mantener una actividad física sistemática, al tiempo que interactúa con otras personas de su edad, que le brindan apoyo social; todo lo cual unido a un sentido de su vida emocional le permita un mejoramiento adecuado" (Martín, 2018).

La aplicación de actividades lúdicas favorece el proceso de socialización entre varios seres cumpliendo estos la función de integración y rehabilitación donde lo principal de este tipo de terapia se enfoca hacia al estudio de individuos los cuales se encuentran pasando por esta fase de madurez; desarrollen habilidades tanto físicas como perceptivas; obteniendo facultades beneficiosas para la salud y a su vez evitando enfermedades graves como osteoporosis, cardiovasculares entre otras.

De esta forma se explica que lo esencial de este tratamiento es desarrollar técnicas sociales para poder relacionarse con un entorno que se torna a veces desconocido. En muchos casos emocionales, puesto que se transmiten sensaciones y sentimientos mientras se realiza la práctica; posibilitando una atención eficiente y específica en ellas, estimulando la creatividad y refuerzo hacia la memoria, llegando a una sensación de bienestar psicofísico profundo debido que, en algunas edades, la mente no funciona como siempre lo ha hecho o como se supone que debe hacerlo.

"El juego constituye una necesidad de gran relevancia integral, puesto que a través de él se adquieren conocimientos, habilidades y sobre todo, le brinda la oportunidad de conocerse así mismo, a los demás y al mundo que los rodea" (Posligua, 2017); es decir que la estabilidad afectiva con la que llegan las personas a la vejez tampoco es igual en todos los individuos sino que va a depender de las relaciones interpersonales que han mantenido y de la propia sensación de haber disfrutado de la vida.

A parte de fijar la construcción de lazos afectivos de forma satisfactoria y saludable; el juego posibilita la relajación por lo que ahuyenta el estrés, la depresión, la ansiedad y todo tipo de emociones negativas.; es aquí donde "el sujeto descubre la capacidad de transformar la realidad por medio de juegos creadores, empleo de símbolos y representaciones" (Stefani, 2014).

"El paradigma de la actividad para el logro de un envejecimiento activo y "bueno", no solo puede apuntar a disminuir el cargo social de adultos mayores enfermos o discapacitados; también apunta al sentido subjetivo del mismo" (Muñoz, 2013) . De acuerdo a lo mencionado la parte socio afectiva del desarrollo integral de los seres humanos; desde la mirada del enfoque cognitivo y sistémico, supone el fortalecimiento de una serie de competencias necesarias para su crecimiento personal y social, las cuales se relacionan con la identificación y control de las propias emociones, el reconocimiento de los aspectos más relevantes del comportamiento humano.

La parte socio-afectiva es un aspecto importante desde una etapa temprana; la interconexión que se crea entre dos personas o más llegan a generar un impacto positivo o negativo en la persona, según sea el caso. Mediante las actividades lúdicas en adultos mayores se busca rehabilitar esas destrezas motrices del sistema sensorial; así como la estimulación cognitiva de la memoria la cual Favorecerá el encuentro intergeneracional para compartir experiencias y recuerdos, como lograr el apoyo y facilitar la relación con la familia.

Los beneficios de estas rutinas lúdicas mantendrán su mente ocupada, haciéndolos sentirse parte de un grupo, estimulando comunicación, aprendizaje y memorización de tareas nuevas u olvidadas alcanzando autoconfianza en sí mismos demostrando como resultados nuevos conceptos. Muchos terapeutas ven a la lúdica como una estrategia de estimulación determinante para fomentar valores, reforzar conocimientos a nivel psíquico, físico y motor mediante la asociación de ideas facilitándole de esta forma la adquisición de capacidades que fortalezcan su proceso de maduración. 
Juan Enrique Tomala Santana, , ,..

Una persona considerada de la tercera suele poseer una menor reserva funcional y una mayor fragilidad en todo en su cuerpo, por ello especialistas de la salud deberán tener los conocimientos y la experiencia necesaria para mejorar las capacidades de este grupo de población; la interconexión que surge entre paciente y médico debe ser completamente mutua para su cuidado, logrando mantener en él la agilidad de mejorar su estabilidad de vida cuya evolución está condicionada por factores psíquicos o sociales.

Cabe aclarar que el juego es un recurso activo donde los ancianos tienen un desenvolvimiento coordinado y balanceado posibilitando el acceso a la creación de nuevas palabras y encontrando solución a problemas y desafíos; el sentido del humor tiene una relación fundamental con el emocional ya que también el juego permite poner en práctica la empatía y comprensión; por lo tanto se deduce que "el sentido del humor tiene una función reparadora; alivia la tensión emocional actuando de purgante psicológico que nos libera temporalmente " (Labarca, 2012).

Ejercicios como la musicoterapia y dibujoterapia hacen que el individuo reestablezca la capacidad respiratoria, el estado de ánimo y aumento de energía corporal. Por ello la flexibilidad aplicada en cada ejercicio tiene como resultado una mayor amplitud y agilidad mental a la hora de realizar algún movimiento; cuando el practicante allá alcanzado dominio de su cuerpo significa que la evolución cognitiva ha mejorado.

Finalmente se puede exponer que la lúdica engloba diversas dimensiones de la vida del adulto mayor tales como: la salud, el juego, la estética, la integración, aprendizaje, las cuales permiten asumir un lugar e identidad, además de un modo de ser particular incidiendo positivamente en el aspecto tanto físico como psicomotriz de la gente que goza de aquel beneficio proporcionado por las actividades lúdicas.

DESARROLLO

\section{MATERIALES Y MÉTODOS}

El trabajo de investigación se efectuó a los adultos mayores del hogar san José para obtener principales patrones de la actividad lúdica, la recolección de información se basó en la aplicación de los siguientes métodos:

\section{Del nivel teórico:}

Histórico - lógico: se usó con el propósito de interpretar el objeto de estudio, su interacción como un todo cuya finalidad es demostrar lo esencial de la bibliografía consultada.

Análisis - Síntesis: Estos métodos diagnosticaron cuales son los tipos de actividades primordiales para la óptima calidad de vida en adultos mayores.

Inductivo - Deductivo: se utilizó para establecer la relevancia del proceso activo de la actividad lúdica en el bienestar físico e intelectual de los individuos tomados como muestra.

\section{De nivel empírico:}

La observación: permitió conocer las características físicas, el contexto donde se encuentran los adultos y la implementación de las actividades lúdicas para producir nuevos cambios en la integración social.

Encuesta: brindo criterios significativos referentes al tipo de actividad empleada en la población seleccionada especificando cuales son las más utilizadas.

La medición: mediante esta se constató el grado de interrelación existente entre los adultos mayores del lugar.

Revisión bibliográfica: fue usada para la recopilación de información relacionada con el tema, a través de libros, de revistas del ámbito científico internet entre otros.

\section{RESULTADOS}


El análisis e interpretación de resultados podrá indicar la influencia de las actividades lúdicas, cuales son los ejercicios o técnicas más usados que favorecen al aspecto físico e intelectual de los adultos mayores en el hogar San José, situado en la ciudad de Guayaquil.

Para esta evaluación y obtención de respuestas se involucró como objeto de estudio a las personas de 65 a 80 años calculando un total de 75 individuos; mediante la tabulación de la encuesta se clasificaron los resultados contabilizando los ejercicios mediante categorías. A continuación se describen las preguntas ejecutadas dentro de la encuesta haciendo uso de tablas y gráficos.

1.- ¿Considera usted que el principal beneficio de las actividades lúdicas es fortalecen la motricidad del cuerpo a nivel físico y mental?

Tabla 1 Actividad lúdica como principal beneficio de la motricidad del cuerpo

\begin{tabular}{lcc} 
Descripción & Frecuencia & Porcentaje \\
\hline Posiblemente si & 22 & $29 \%$ \\
\hline Si & 48 & $64 \%$ \\
Posiblemente no & 5 & $7 \%$ \\
No & 0 & $0 \%$ \\
Total & $\mathbf{7 5}$ & $\mathbf{1 0 0 \%}$
\end{tabular}

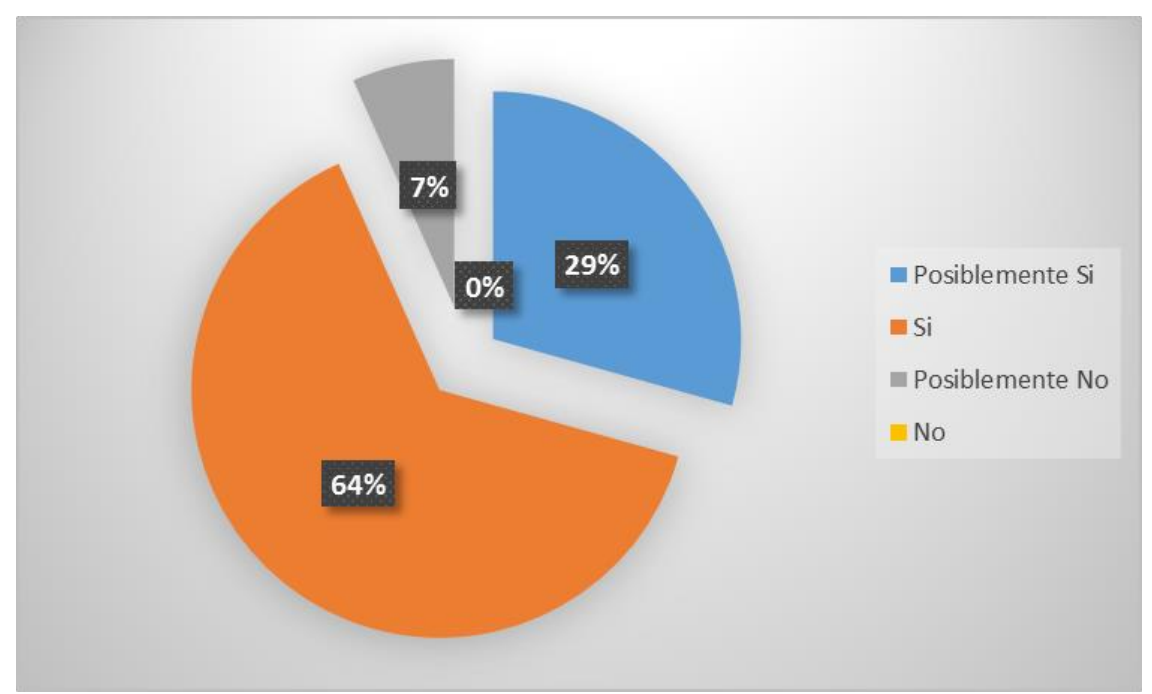

Gráfico 1 Porcentajes obtenidos referente a la pregunta 1

Fuente: Elaboración propia del autor

La ludica no se limita a la edad, lo esecial es que el individuo adapte sus necesidades y propósitos por lo general; aquellos que no están en buena forma física tienen que esforzarse más.La mayor parte de la actividad ejecutada en este tipo de seres debe ser aeróbica y de intensidad moderada durante 3 dias por semanas para que los huesos y músculos incrementen alguna cualidad como la coordinación, la elasticidad o la flexibilidad .De acuerdo a la informacion recopilada dentro del Hogar San Jose describe que el 64\% de los adultos mayores afirman un mayor porcentaje hacia la opcion "Si"; especificando que si fortalecen la motrocidad del cuerpo a nivell fisico y mental las actividades ludicas, a diferencia de las otras opciones las cuales poseen una proporción baja referente a la pregunta efectuada.

2.- ¿Piensa usted que las actividades lúdicas promueven la integración social y el autoaprendizaje en los adultos?

Tabla 2 La lúdica promueve la integración social y autoaprendizaje 


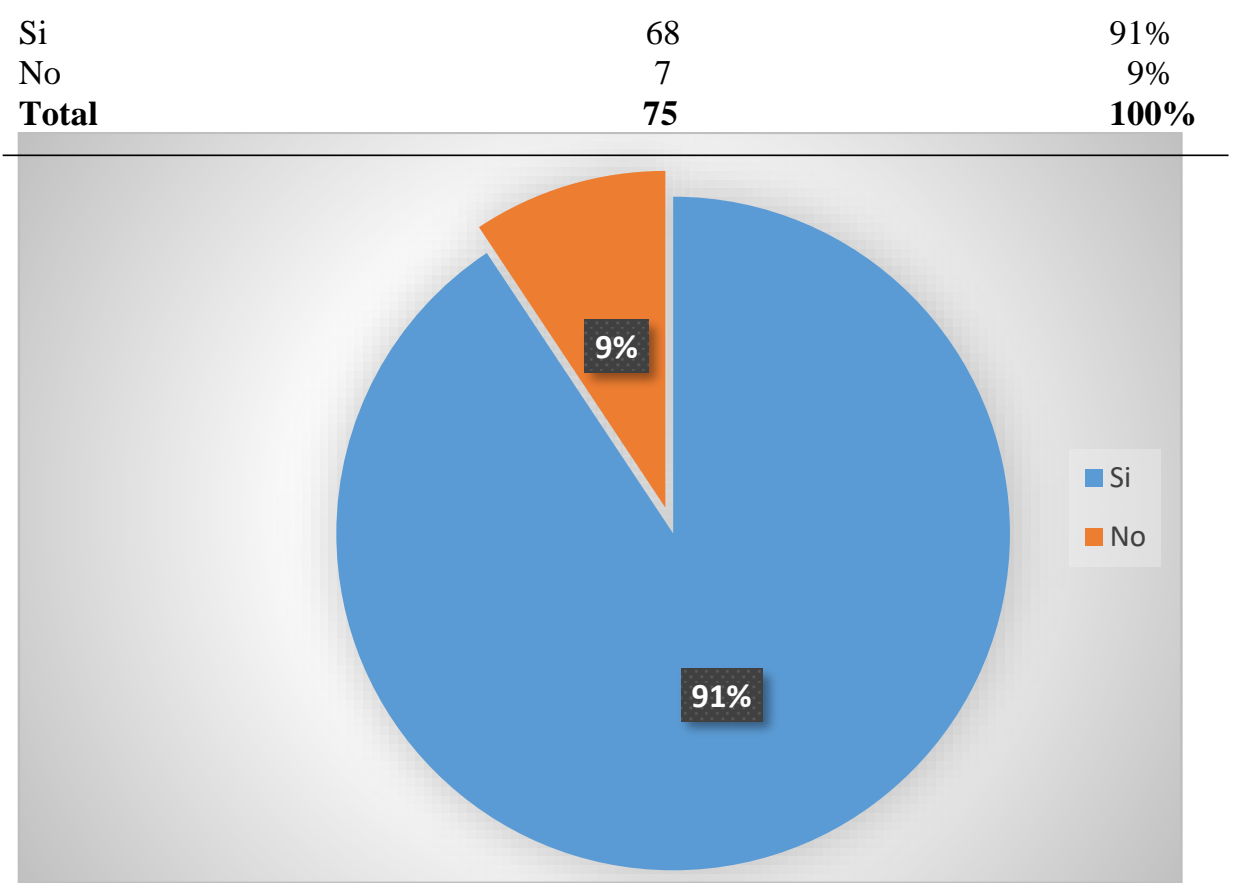

Gráfico 2 Respuestas obtenidas referente a la pregunta 2

Fuente: Elaboración propia del autor

Según los encuestados el $91 \%$ dicen que "Si" a través de la práctica lúdica se promueve la integración social y el autoaprendizaje llegando a coincidir con los estudios del psicólogo Barrera donde menciona que "el mayor puede ejercer (o no) su sociabilidad en muy distintos espacios y entornos de la ciudad tomando en cuenta las distintas perspectivas en la que los espacios se pueden hacer efectivos para la integración" (Barrera, 2010); por ello los especialistas consideran al juego "elemento primordial", el cual permite expresar a través de actividades que uno tiene en mente durante el desarrollo de destrezas didácticas aplicando un correcto manejo tendrá un significado concreto y positivo para el mejoramiento del aprendizaje en cuanto a la cualificación, formación crítica, valores, relación y conexión con los demás.

3.- ¿cuáles son los tipos de actividades que usted realiza mayormente para reforzar y mantener la memoria activa?

Tabla 3 Tipos de actividades lúdicas

\begin{tabular}{lcc} 
Descripción & Frecuencia & Porcentaje \\
\hline Juego de naipes & 6 & $8 \%$ \\
El bingo & 8 & $11 \%$ \\
Rompecabezas & 10 & $13 \%$ \\
Juego de pelotas & 4 & $5 \%$ \\
Dibujoterapia & 24 & $32 \%$ \\
Sopa de letras & 20 & $27 \%$ \\
La galleta & 3 & $4 \%$ \\
Total & $\mathbf{7 5}$ & $\mathbf{1 0 0 \%}$ \\
\hline
\end{tabular}




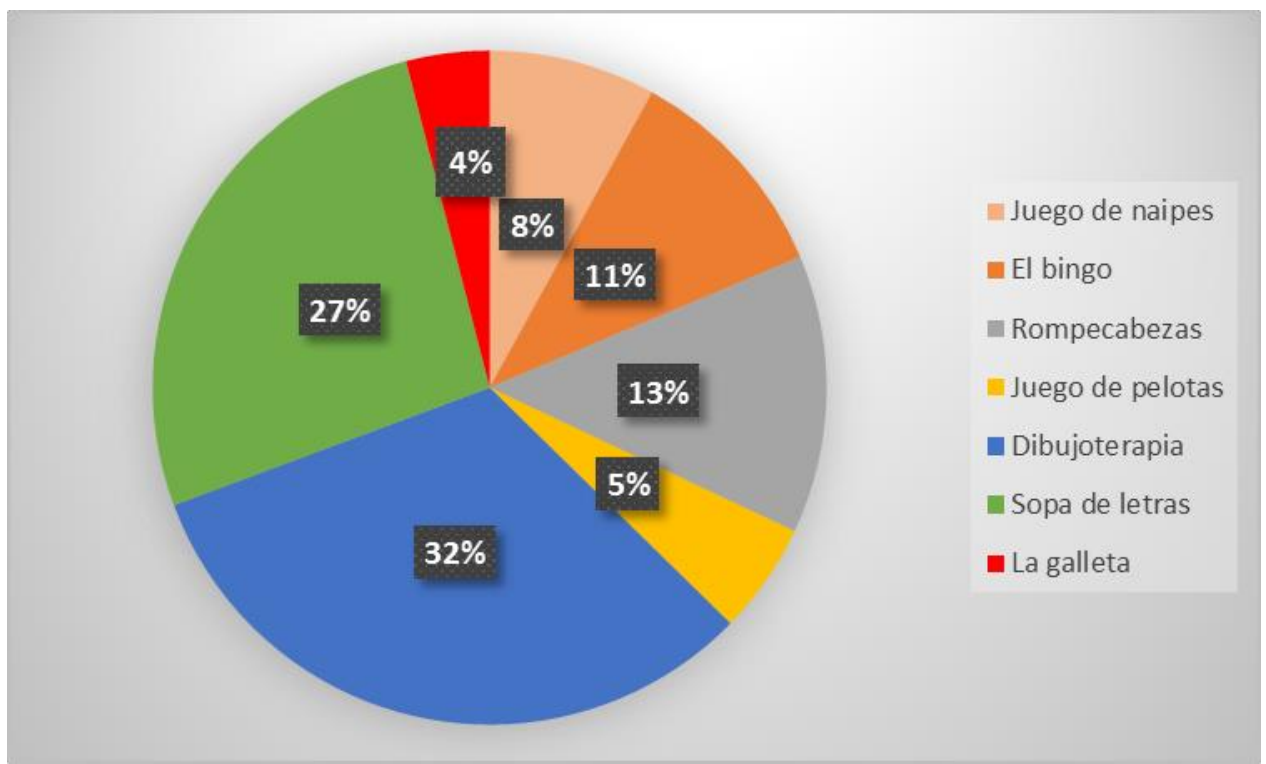

Gráfico 3 Porcentajes obtenidos referente a la pregunta 3

Fuente: Elaboración propia del autor

En la tabla se observan los distintos porcentajes a cerca de las actividades mencionadas donde los adultos mayores establecen que la dibujoterapia (32\%) y las sopas de letras (27\%) son las técnicas más utilizadas dentro del lugar para mantener la correcta estabilidad de la memoria De acuerdo a investigaciones de científicos de la universidad de Boston mencionan dentro de un articulo del The New York Time "el entrenamiento cognitivo actúa como un director de orquesta, al escuchar, sintetizar y dirigir; mejora del desarrollo, mantenimiento cognitivo a un nivel normal o prevención del declive asociado a la edad" (Carey, 2019).Las otras actividades mencionadas cumplen la misma acción de potenciar la memoria; además de ser beneficiosas a nivel cognitivo, lo son también a nivel emocional; son agilidades en la que los ancianos se divierten, pasan un buen rato de relajación .

4.- ¿considera necesaria la prevención y tratamiento de enfermedades mediante la práctica de actividades lúdicas, durante el envejecimiento de los adultos mayores?

Tabla 4 Prevención y tratamiento de enfermedades mediante las actividades lúdicas.

\begin{tabular}{lcc} 
Descripción & Frecuencia & Porcentaje \\
\hline $\mathrm{Si}$ & $\mathbf{7 2}$ & $96 \%$ \\
No & 3 & $4 \%$ \\
Total & $\mathbf{7 5}$ & $\mathbf{1 0 0 \%}$ \\
\hline
\end{tabular}




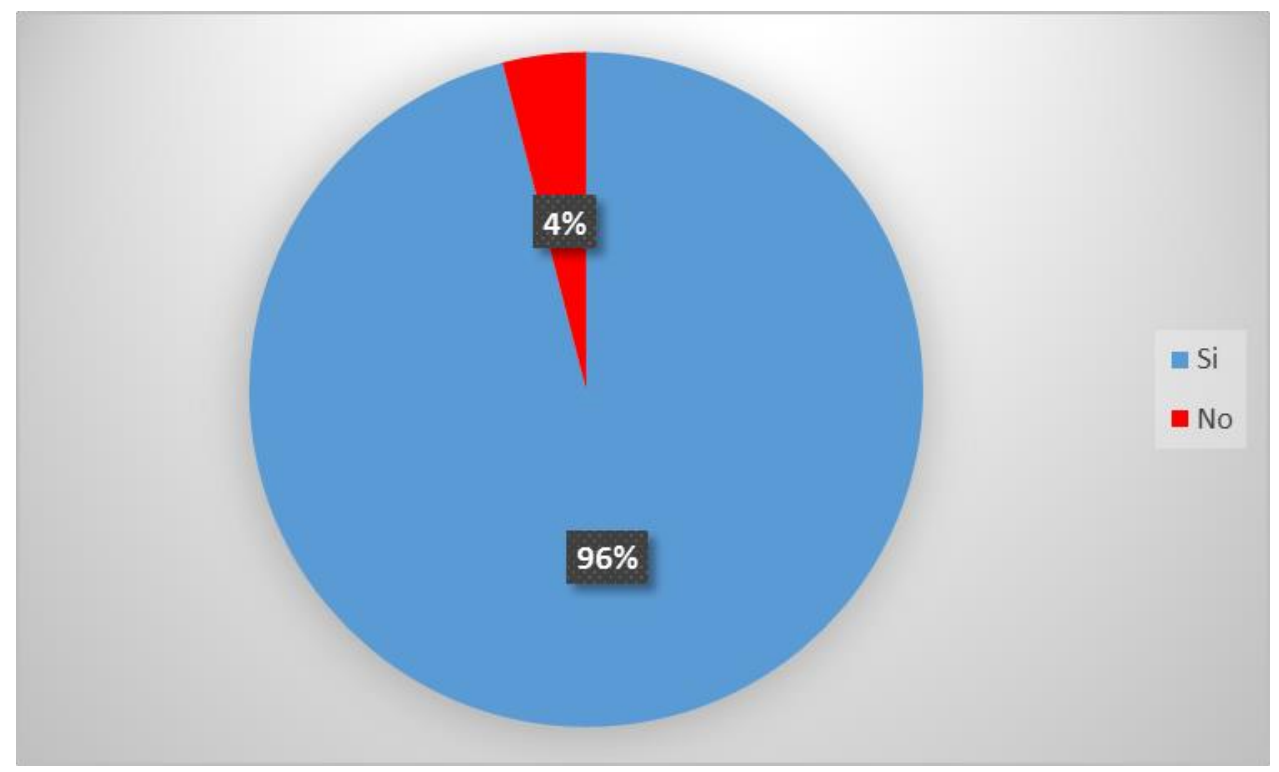

Gráfico 4 Respuestas obtenidas referente a la pregunta 4

Fuente: Elaboración propia del autor

El trabajo de la lúdica reduce los factores de riesgo contra enfermedades, cuando se realiza con regularidad, la actividad aeróbica moderada e intensa genera mayor circulación de sangre hacia los músculos y las concentraciones de oxígeno en la sangre aumentan. De acuerdo al grafico se indica que el $96 \%$ de la población consideran la práctica de actividades lúdicas como una herramienta de prevención y tratamiento de enfermedades que puedan afectar la salud de los adultos mayores durante la etapa del envejecimiento; sin embargo, el $4 \%$ señala que no son necesarias.

\section{DISCUSIÓN}

Dentro de otros estudios enfocados en la temática de actividades lúdicas se obtienen múltiples resultados a cerca del beneficio proporcionado por la lúdica durante el envejecimiento como en el caso del artículo titulado Actividades recreativas en adultos mayores internos en un hogar de ancianos cuyo contenido especifica que estos ejercicios pueden mejoran la comunicación la flexibilidad del cuerpo, la expresión y la socialización con su entorno; así como también la prevención de enfermedades que pongan en riesgo la salud del individuo. Según el autor del texto "una persona mayor con una autoestima frágil puede interpretar expresiones de aliento bien intencionadas; el envejecimiento tiene repercusión en todas las etapas de la vida y más cuando acentúa cualquier factor psicosocial" (Giai, 2015)

En comparación con los resultados obtenidos se deduce un porcentaje elevado sobre el beneficio de las actividades lúdicas pues estas son consideradas como juegos que no solo favorecen a los estímulos del cuerpo humano e integración con el mundo; sino se las puede denominar como estrategias didácticas de como pasar en el tiempo libre; las cuales usan básicamente una metodología simple y sencilla, fáciles de aprender diseñadas para establecer una interconexión motriz de las personas.

La parte lúdica cumple una función social y cultual ya que gracias a estas prácticas los adultos mayores mantienen un estilo de vida activo y productivo adoptándose a nuevos hábitos sin sufrir alteraciones en su bienestar.

\section{CONCLUSIONES}


Según los resultados obtenidos durante el desarrollo del trabajo investigación "Beneficios de las actividades lúdicas en adultos mayores"; el autor llega a las siguientes conclusiones:

- Mejoran la resistencia y la masa muscular; disminuyendo la tensión arterial; prevención de enfermedades como la osteoporosis.

- Favorecen la coordinación de distintos tipos de movimientos.

- Desarrollan destrezas físicas y mentales produciendo confianza en sí mismo y en sus capacidades.

- Permiten el descubrimiento del pensamiento crítico y creativo.

- Promueven las ventajas del aprendizaje activo.

- Potencia la percepción sensorial ampliando los niveles de satisfacción vital.

- Elevan la autoestima, la autoconfianza, la autoimagen y la seguridad.

- Contribuyen a la cultura comunitaria e integración social.

\section{REFERÉNCIAS BIBLIOGRÁFICAS}

Barrera, E. (2010). La integración social de las personas mayores en espacios urbanos. Aposta revista de ciencias sociales, 3(46), 1-22. Obtenido de http://www.apostadigital.com/revistav3/hemeroteca/barrera2.pdf

Carey, B. (17 de Mayo de 2019). La ciencia de la estimulación cerebral. The New York Time. Obtenido de https://www.nytimes.com/es/2019/05/17/espanol/estimulacion-cerebral-memoria.html

Giai, M. (2015). Actividades recreativas en adultos mayores internos en un hogar de ancianos. Revista Cubana de Salud Pública., 41(1), 67-76. Obtenido de http://scielo.sld.cu/

Labarca, C. (2012). Sentido del humor en el adulto mayor. Telos, 14(3), 400-414.

Lúdico. (2019). Significados.com. Obtenido de https://www.significados.com/ludico

Martín, R. (2018). Actividad física y calidad de vida en el adulto mayor. Una revisión narrativa. Revista Habanera de ciencias médicas, 17(5), 813-815. Obtenido de http://www.revhabanera.sld.cu/index.php/rhab/article/view/2418

Muñoz, C. (2013). Bienestar subjetivo y actividad social con sentido histórico en adultos mayores. Hacia la Promoción de la Salud, 18(2), 13-26.

Organización Mundial de la Salud, O. (12 de Diciembre de 2017). La salud mental y adultos mayores. Organización Mundial de la Salud, Ginebra. Obtenido de https://www.who.int/es/news-room/fact-sheets/detail/la-saludmental-y-los-adultos-mayores

Posligua, J. (2017). Incidencia de las actividades lúdicas en el desarrollo del pensamiento creativo. Dominios de la ciencia, 3(3). Obtenido de http://dominiodelasciencias.com/ojs/index.php/es/index

Querales, J. (2011). Influencia de la actividad física recreativa en el estilo de vida del adulto mayor de la comunidad del sector Agua Dulce de la Parroquia Barinitas del Municipio Bolívar . Lecturas: Educación Física y Deportes, 16(161). Obtenido de http://www.efdeportes.com/efd161/actividad-fisica-recreativa-del-adultomayor.htm

Stefani, G. (2014). Transformaciones lúdicas. Un estudio preliminar sobre los tipos de juego y espacios lúdicos. Interdisciplinaria, 31(1), 39-55. Obtenido de 
Juan Enrique Tomala Santana, , ...

178 UNESUM-Ciencias. Publicación cuatrimestral. Vol. 4, Año 2020, No. 1 (Enero - Abril) 